\title{
BMJ Open Gut microbiota composition and arterial stiffness measured by pulse wave velocity: case-control study protocol (MIVAS study)
}

\begin{abstract}
Rita Salvado (D) , ${ }^{1}$ Sandra Santos-Minguez, ${ }^{2}$ Cristina Agudo-Conde, ${ }^{1}$ Cristina Lugones-Sanchez, ${ }^{1}$ Angela Cabo-Laso, ${ }^{1}$ Jesus $\mathrm{M}^{\mathrm{a}}$ Hernandez-Sanchez, ${ }^{2}$ Rocio Benito, ${ }^{2}$ Emiliano Rodriguez-Sanchez, ${ }^{1,3}$ Manuel A Gomez-Marcos (D) ,1,3 Jesus M Hernandez-Rivas, ${ }^{2,4}$ Pedro Guimarães Cunha, ${ }^{5}$ Luis Garcia-Ortiz, ${ }^{1,6}$ MIVAS Investigators ${ }^{7}$
\end{abstract}

To cite: Salvado R, SantosMinguez S, Agudo-Conde C, et al. Gut microbiota composition and arterial stiffness measured by pulse wave velocity: case-control study protocol (MIVAS study). BMJ Open 2021;11:e038933. doi:10.1136/ bmjopen-2020-038933

- Prepublication history for this paper is available online. To view these files, please visit the journal online (http://dx.doi. org/10.1136/bmjopen-2020038933).

Received 29 March 2020 Revised 29 November 2020 Accepted 02 January 2021

Check for updates

(c) Author(s) (or their employer(s)) 2021. Re-use permitted under CC BY-NC. No commercial re-use. See rights and permissions. Published by BMJ.

For numbered affiliations see end of article.

Correspondence to

Dr Rita Salvado;

ritasalvado@usal.es

\section{ABSTRACT}

Introduction Intestinal microbiota is arising as a new element in the physiopathology of cardiovascular diseases. A healthy microbiota includes a balanced representation of bacteria with health promotion functions (symbiotes). The aim of this study is to analyse the relationship between intestinal microbiota composition and arterial stiffness.

Methods and analysis An observational case-control study will be developed. Cases will be defined by the presence of at least one of the following: carotid-femoral pulse wave velocity (cf-PWV), Cardio-Ankle Vascular Index (CAVI), brachial ankle pulse wave velocity (ba or ba-PWV) above the $90^{\text {th }}$ percentile, for age and sex, of the reference population. Controls will be selected from the same population as cases. The study will be developed in Primary Healthcare Centres. We will select 500 subjects ( 250 cases and 250 controls), between 45 and 74 years of age. Cases will be selected from a database that combines data from EVA study (Spain) and Guimarães/Vizela study (Portugal). Measurements: cf-PWV will be measured using the SphygmoCor system, CAVI, ba-PWV and Ankle-Brachial Index will be determined using VaSera device. Gut microbiome composition in faecal samples will be determined by 16 S ribosomal RNA sequencing. Lifestyle will be assessed by food frequency questionnaire, adherence to the Mediterranean diet and IPAQ (International Physical Activity Questionnaire). Body composition will be evaluated by bioimpedance.

Ethics and dissemination The study has been approved by 'Committee of ethics of research with medicines of the health area of Salamanca' on 14 December 2018 (cod. 2018-11-136) and the 'Ethics committee for health of Guimaraes' (Portugal) on 15 0ctober 2019 (ref: 67/2019). All study participants will sign an informed consent form agreeing to participate in the study, in compliance with the Declaration of Helsinki and the WHO standards for observational studies. The results of this study will allow a better description of gut microbiota in patients with arterial stiffness.

Trial registration details ClinicalTrials.gov, identifier NCT03900338
Strengths and limitations of this study

- Observational multicentric and multi-country case-control study evaluating the relationship of gut microbiota and arterial stiffness, in 500 subjects from 40 to 74 years of age.

- Two hundred and fifty cases were defined by the presence of at least one of the following: carotidfemoral pulse wave velocity, Cardio-Ankle Vascular Index or brachial ankle pulse wave velocity above the $90^{\text {th }}$ percentile, for age and sex, of the reference population. Cases will be matched with 250 controls using propensity score.

- Analyses of the composition of the gut microbiome in faecal samples by $16 \mathrm{~S}$ ribosomal RNA sequencing.

- It is not a random sample, and therefore it cannot be said that the representation is population-based.

- Some of the drugs taken by the included subjects could modify the microbiota.

\section{INTRODUCTION}

Cardiovascular diseases (CVD) are the leading cause of morbidity and mortality globally. ${ }^{1}$ In the latest years, a big effort has been made to improve the identification of individuals at high risk of suffering a cardiovascular event, looking beyond classical risk factors, using biomarkers that reflect early functional or morphological changes, before overt disease manifests, allowing timely treatment of subclinical disease. ${ }^{2}$

Arterial stiffness has been proven to have a good predictive value for CVD, ${ }^{3}$ in various populations, with different levels of risk: general population, elderly, patients with type 2 diabetes, hypertension or end-stage renal disease. ${ }^{4}$ Arterial stiffness reflects the aortic wall damage caused by several cardiovascular risk factors, over a long period of 
time, signalling the patients in which arterial risk factors were translated to real risk. ${ }^{5}$ There are several methods to measure arterial stiffness. Carotid to femoral pulse wave velocity (cf-PWV) is the gold standard, ${ }^{6}$ others widely accepted are: Cardio-Ankle Vascular Index (CAVI), which measures the stiffness of the aorta, femoral artery and tibial artery, ${ }^{7}$ brachialankle pulse wave velocity (ba-PWV), which uses brachial and tibial arterial waves. ${ }^{8}$

The evaluation of carotid intima-media thickness (IMT) can identify the presence of atherosclerotic plaques which traduces structural damage .

The major determinants of arterial stiffness are age and hypertension but gender and classical cardiovascular risk factors also play an important role. ${ }^{5}$ Other factors, as genetic burden, systemic inflammatory diseases and gut microbiota, ${ }^{9}$ have also been linked to pulse wave velocity. Gut microbiota composition has also been implicated on the genesis of hypertension, ${ }^{10}$ obesity ${ }^{11}$ insulin resistance, metabolic syndrome ${ }^{11}$ and type 2 diabetes. ${ }^{12}$

Gut microbiota is a new player in the pathophysiology of cardiovascular disease. There are 100 trillion bacteria in the human gut, with 3.3 million non-redundant genes, one hundred times the human genome, which gives human microbiome a huge metabolic potential. In adult's gut microbiota, the majority of the microbial populations belong to the bacteria domain, with approximately $90 \%$ of Bacteroidetes and Firmicutes phyla. Commensal gut microbiota has two main functions: intervenes in human immunological response and contributes to energy harvest from non digestible starches.

The shift from a healthy microbiota toward dysbiosis mean that there is an increase in pathobionts and is likely to be triggered by environmental factors. ${ }^{11}$ Although age and gut's genetically defined architecture are the most relevant factors influencing gut's microbiome composition, ${ }^{13}$ diet and lifestyle are likely the major causes of inter-individual variation in the composition of human gut's microbiome. There is evidence that the consumption of artificial sweeteners, ${ }^{14}$ dietary emulsifiers, ${ }^{15}$ a highsalt $\operatorname{diet}^{16}$ and obesity ${ }^{17}$ alter the gut microbiota, reduce microbial diversity and induce inflammation, whereas a diet rich in vegetables has been linked to a healthy microbial diversity. ${ }^{18} 19$

Dysbiosis is characterised by a greater amount of proinflammatory species, that favour metabolic diseases development, caused by both diet-dependent and independent mechanisms. ${ }^{11}{ }^{20}$ Diet independent mechanisms are mediated by two major receptor families that detect microbes: Toll-like receptors and Nod-like receptors, which sense the presence of intracellular microbes. ${ }^{21}$ The activation of these receptors triggers inflammatory reactions in the liver, white adipose tissue, brain and other organs, and triggers metabolic disorders, such as insulin resistance. $^{22}$

Diet-dependent mechanisms result from microbial enzymatic activities. Some are beneficial, like microbial fermentation of polysaccharides, producing short-chain fatty acid (SCFA) and bile-acid. Others are detrimental, such as phosphatidylcholine metabolisation by intestinal microorganisms, that results in the production of trimethylamine N-oxide, which is associated with cardiovascular disease development and progression. ${ }^{23-25}$

Our hypothesis is that patients with arterial stiffness have a different intestinal flora, when compared with healthy controls, i.e, subjects free from cardiovascular disease. We also hypothesise that gut microbiota will be different in subjects with different lifestyles and body composition, as well as with target organ damage and neurocognition.

The main objective of this study will be to analyse the relationship between intestinal microbiota composition and arterial stiffness in a population without cardiovascular disease.

As secondary objectives we will consider the relationship of gut microbiota with other measures of vascular structure and function, end organ disease, cognition, cardiovascular risk factors, body composition and lifestyles. We will also analyse gender differences in intestinal microbiota composition and its relationship with vascular structure and function.

\section{METHODS AND ANALYSIS \\ Design and setting}

This is an observational, multicentric, case-control study. Cases will be defined by the presence of at least one of the following: cf-PWV, CAVI or ba-PWV above the $90^{\text {th }}$ percentile, for age and sex, of the reference population. It will be an international study including patients from two neighbour countries: Portugal, through the Life and Health Sciences Research Institute, Minho University (Braga) and Spain, through the Primary Care Research Unit of Salamanca (APISAL) belonging to the Biomedical Research Institute of Salamanca (IBSAL) and Cancer Research Institute of Salamanca (CIC) that will also perform gut microbiota analysis.

\section{Study population}

We will select 500 subjects, aged 45 to 74 years, free of cardiovascular disease. We will recruit patients from a database that combines data from EVA study ${ }^{26}$ (Spain) and Guimarães/Vizela study ${ }^{27}$ (Portugal), and if necessary, we will incorporate new patients who meet the inclusion criteria to complete the sample (figure 1). Controls will be selected from the same population as cases. The methodology of these studies was published elsewhere. ${ }^{2627}$

\section{Selection criteria}

Inclusion criteria: Patients between 45 and 74 years old, who agree to participate in the study and do not meet any of the exclusion criteria.

Exclusion criteria: History of CVD (ischaemic heart disease or stroke, peripheral arterial disease or heart failure), diabetes mellitus, renal failure in terminal stages (glomerular filtration rate below 30\%), chronic inflammatory diseases, inflammatory bowel disease, body mass index $>40 \mathrm{~kg} / \mathrm{m}^{2}$, oncological disease diagnosed in the 


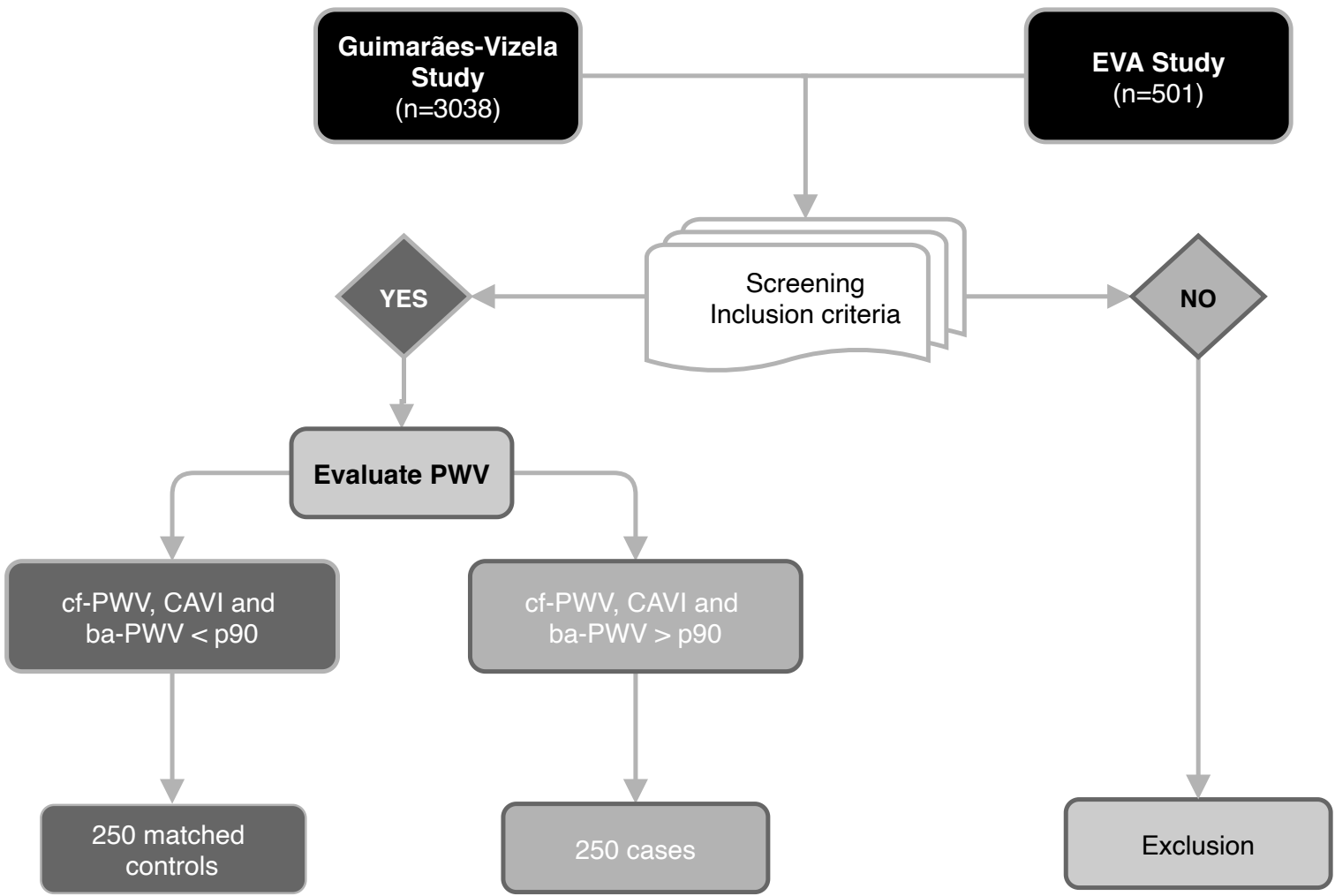

Figure 1 Study flow chart. cf-PWV,carotid-femoral PWV; CAVI, Cardio-Ankle Vascularlndex; ba-PWV, brachial-ankle PWV; PWV, pulse wave velocity.

last 5 years and/or under treatment, terminal conditions, antibiotic use within the last 15 days and those who refuse to sign the informed consent.

\section{Sample size}

The sample size was estimated to detect a minimum OR of 1.75 in the study factor (microbiota dysbiosis), considering vascular stiffness as a dependent variable and accepting an $\alpha$ risk of 0.05 and a $\beta$ risk of 0.20 , in a two-sided test, assuming a rate of losses due to technical difficulties or refusal to participate of $5 \%$, and a rate of exposure of $0.3 \%$, in the control group. Therefore, it will be necessary to include 500 subjects, 250 with arterial stiffness and 250 controls.

\section{Patient and public involvement}

Patients will not participate in the study design, however they will actively participate in the study recruitment by disseminating the study objectives and inclusion criteria through their organisations. At the end of the study, in addition to sending a detailed report with the results of each patient, a dissemination session will be organised for all patients included in the study. Some of the participants will take part in this conference to share their experience and their personal evaluation of the study results.

\section{Variables and measurement instruments}

General and potentially effect-modifying variables such as age, gender, occupation, family history of CVD, hypertension, dyslipidaemia, hypothyroidism and drug use will be documented.

\section{Anthropometric measurements}

Body weight will be measured twice, using a homologated electronic scale (Seca 770 medical scale and measurement systems, Birmingham, UK) after calibration (precision $\pm 0.1 \mathrm{~kg}$ ), with the patient wearing light clothing and barefoot. Body mass index (BMI) will be calculated as weight $(\mathrm{kg})$ divided by height squared $\left(\mathrm{m}^{2}\right)$. Waist circumference will be measured using a flexible graduated measuring tape, with the patient in the standing position, without clothing.

Body composition will be determined by the InBody 230 monitor (InBody Co, Ltd, Seoul, Korea), which gives information of body composition analysis.

Office or clinical blood pressure (BP) will involve three measurements of systolic BP (SBP) and diastolic BP (DBP), using the average of the last two, with a validated OMRON model by following the recommendations of the European Society of Hypertension (ESH) ${ }^{28}$

\section{Habits and lifestyles}

\section{Diet}

Adherence to the Mediterranean diet, will be measured using the validated 14-point Mediterranean Diet Adherence Screener (MEDAS), ${ }^{29}$ developed by the PREDIMED study group. Each question will be scored as 0 or 1. Adequate adherence to the Mediterranean diet will be assumed when the total score is $\geq 9$ points.

With the application developed in the EVIDENT study ${ }^{30}$ (registry number 00/2014/2207), food consumption will be recorded during a usual week. 
The dietary habits of participants will be assessed using a semi-quantitative 137-item Food-Frequency Questionnaire (FFQ), previously validated in Spain $^{31}$ and Portugal. ${ }^{32}$ The FFQ is based on typical portion sizes that will be multiplied by the consumption frequency for each food. This estimated frequency refers to the previous year, from the time of the interview, and is divided into nine intake frequency categories ranging from never to more than six servings per day.

\section{Physical activity}

Two questionnaires will be used to assess physical activity:

International Physical Activity Questionnaire-Short Form $(I P A Q-S F)$. The short form (nine items) categorises physical activity for the last 7 days in three levels of intensity: (1) intense physical activity, (2) moderate-intensity activity and (3) light activity. ${ }^{33}$

Questionnaire hours seated (Marshall): Measures the amount of time spent sitting, at work, in the displacements and at home, during week days and weekend. ${ }^{34}$

\section{Tobacco and alcohol consumption}

Standardise questionnaire will be used to assess tobacco and alcohol use.

\section{Laboratory measurements}

Venous blood samples will be taken between 08:00 and 09:00 a.m., after participants have fasted and abstained from smoking, alcohol and caffeinated beverages consumption, for 12 hours. Fasting plasma glucose, creatinine, uric acid, liver function and lipids levels will be measured using standard enzymatic automated methods. A blood sample of each participant will be frozen for posterior evaluation of total biliary acids, deoxycholic acid and SCFA concentration $(\mathrm{mg} / \mathrm{mL})$.

\section{Gut microbiota measurements}

Stool samples will be collected by participants with the OMNIgene GUT (OMR -200) kit that permits transport and store with preservation of stabilised DNA, at room temperature, for 60 days. This kit allows obtaining high quality DNA suitable for $16 \mathrm{~S}$ ribosomalRNA (rRNA) microbiome profiling and ensures microbiota profiles accurately represent the in vivo state. All specimens will be sent to the Cancer Research Center in Salamanca.

DNA will be extracted using the FastDNA Soil method, according to the manufacturer's instructions (FastDNA SPIN Kit for Soil (MP Biomedicals, USA)). DNA will be extracted from 100 to $150 \mu \mathrm{l}$ of different faeces samples. Each sample will be added to a Lysing Matrix E tube (each tube contains $1.4 \mathrm{~mm}$ ceramic spheres, $0.1 \mathrm{~mm}$ silica spheres and one $4 \mathrm{~mm}$ glass beads) and mixed with different buffers to solubilise membrane proteins, extracellular proteins and contaminants in the samples. Afterwards, the sample mix will be homogenised in the Cell disrupter Thermo Savant Fastprep FP120 at $6 \mathrm{~m} / \mathrm{s}$ for $40 \mathrm{~s}$. This allows a mechanical disruption of cell walls of the present organisms. DNA will then be eluted with $50 \mu \mathrm{l}$ of DNase/Pyrogen-Free Water (DES), after successive washing steps through the silica Binding Matrix where the purified nucleic acids were retained. Finally, purified DNA will be measured in the NanoDrop, to check quality and quantity.

DNA yield (ng $\mu \mathrm{l}-1$ ) will be quantified spectrophotometrically with a NanoDrop 2000c Spectrophotometer (Thermo Fisher Scientific, USA). It will also be quantified fluorometrically in a TapeStation 2200, using Genomic DNA ScreenTapes (Agilent, USA) and in a Qubit 4.0 fluorometer (Invitrogen, USA). DNA extraction efficiency will be calculated as the total amount of DNA extracted per biomass ( $\mu$ g g wet wt -1 ). NanoDrop will also be used to estimate the purity of the extracted DNA. Low absorption ratios at $260 / 280 \mathrm{~nm}(<1.7)$ will be used as an indicator of protein impurities, and low absorption ratios at $260 / 230 \mathrm{~nm}(<2)$ will be used as an indicator of contamination from polysaccharides. The TapeStation system performs electrophoresis in so-called ScreenTapes and outputs images of DNA integrity as well as a DNA Integrity Number (DIN) based on the sizes of the isolated DNA. The DIN ranges from 1 to 10 , and a high DIN indicates large DNA fragments, whereas a low DIN indicates more fragmented DNA. DIN determines the fragmentation of a genomic DNA sample by assessing the distribution of signal across the size range using a proprietary algorithm.

\section{Amplicon and Illumina sequencing of bacterial 16S rRNA genes}

Amplicon sequences of bacterial 16S rRNA genes, which target v3 to v4 regions, will be obtained using Illumina predesigned primer pair, as previously described: ${ }^{35}$

S-D-Bact-0341-b-S-17, 5'CCTACGGGNGGCWGCAG-3' and S-D-Bact-0785-a-A-21, 5'-GACTACHVGGGTATCT $A A T C C-3$ ', with a length of $465 \mathrm{bp}$.

Since v5 to v6 regions have also been considered the most functional regions, together with $\mathrm{v} 4,{ }^{36}$ and two of the most relevant regions for phylogenetic classification, we will also analyse these regions. The v5 to v6 primers were obtained from ${ }^{37}$ being the sequences: $\mathrm{V}_{5} \mathrm{~F}_{-}$ Nextera 5'- RGGATTAGATACCC-3' and V6R_Nextera 5'CGACRRCCATGCANCACCT-3', with a length of $281 \mathrm{bp}$. Both primers pair, targeting the variable regions v3 to v4 and v5 to v6 are equipped with Illumina adapter overhang nucleotide sequences:

- Forward overhang: 5' TCGTCGGCAGCGTCAGATGT GTATAAGAGACAG-(locus-specific sequence).

- Reverse overhang: 5' GTCTCGTGGGCTCGGAGATG TGTATAAGAGACAG-(locus-specific sequence).

The resulting amplicons will be purified using Agencourt AMPure XP (Beckman Coulter) as recommended by the manufacturer. They will then be amplified in a second PCR where the indexes will be added. The index is unique for each sample of each patient. Once the samples are indexed and identified, the last purification using the Agentcourt AMPure XP kit will be performed. At this point, amplicon libraries of each sample are generated. These libraries should be quantified in the Qubit, normalised and pooled in equimolar amounts. The pooled samples will be sequenced, using an Illumina 
MiSeq with 2×300 bp v3 chemistry, to obtain high quality scores.

\section{Bioinformatics}

Raw sequence data will be analysed using an in-house pipeline. This pipeline includes base pair quality filtering, alignment and comparison to a reference database. Quality passing-filter readings will be clustered into operational taxonomic units. Then, we will compare control versus cases samples. All the microbiome differences will be then interpreted and classified. Quality control will be carried out on a per sample basis, discarding pairedends with an overlap of less than 200nt and removing chimeric sequences using de novo chimaera detection in USEARCH. $^{38}$

\section{Vascular structure and function \\ Carotid-femoral pulse wave velocity and Central Augmentation Index}

These parameters will be estimated using the SphygmoCor System (AtCor Medical Pty Ltd, Head Office, West Ryde, Australia). With the patient sitting and resting his/her arm on a rigid surface, pulse wave analysis will be performed with a sensor in the radial artery, using a mathematical transformation to estimate the aortic pulse wave. CentralAugmentation Index (CAIx) will be estimated from aortic wave morphology using the following formula: increase in central pressure $\times 100 /$ pulse pressure, and it will be adjusted for a heart rate of $75 \mathrm{bpm}$ (beats per minute). Carotid and femoral artery pulse waves will be analysed, with the patient in a supine position, using the SphygmoCor System, estimating the delay, as compared with the ECG wave, and calculating cf-PWV. Distance measurements will be taken with a measuring tape from the sternal notch to the carotid and femoral arteries at the sensor location and will be multiplied by 0.8 . Subclinical organ damage will be defined as cf-PWV, above the $90^{\text {th }}$ percentile, for age and sex, of the reference population. $^{39}$

\section{Cardio-Ankle Vascular Index, brachial ankle PWV and Ankle Brachial Index}

These parameters will be estimated using the VaSera device VS-2000 (Fukuda Denshi Co, Ltd, Tokyo, Japan). CAVI values will be automatically calculated by substituting the stiffness parameters in the following equation to detect the vascular elasticity and the cardio ankle PWV: stiffness parameter $\beta=2 \rho \times 1 /(\mathrm{Ps}-\mathrm{Pd}) \times \ln (\mathrm{Ps} / \mathrm{Pd}) \times \mathrm{PWV}^{2}$ where $\rho$ is the blood density, Ps and Pd are SBP and DBP in $\mathrm{mmHg}$, and $\mathrm{PWV}$ is measured between the aortic valve and ankle. The average coefficient of the variation of CAVI is $<5 \%$, which is small enough for clinical use and confirms that CAVI has favourable reproducibility. ${ }^{40}$ The ba-PWV will be estimated using the following equation: ba-PWV $=((0.5934 \times$ height $(\mathrm{cm})+14.4724)) / \mathrm{tba}$, where tba is the time the same waves were transmitted to the ankle. ${ }^{41}$ For this study, the mean AnkleBrachial Index (ABI), CAVI and ba-PWV obtained will be considered.
CAVI will be classified as: normal $(\mathrm{CAVI}<8)$, borderline $(8 \leq \mathrm{CAVI}<9)$ and abnormal $(\mathrm{CAVI} \geq 9) .{ }^{42}$ Subclinical organ damage will be defined, CAVI or ba-PWV above the $90^{\text {th }}$ percentile of the reference population. ${ }^{39} \mathrm{ABI} \leq 0.9$ will be considered abnormal. ${ }^{2}$

\section{Central and peripheral augmentation index by the wrist-worn} device

Participants will be examined in a seated position, after 10 min of rest with his/her arm supported on a firm surface, at heart height. The wrist-worn device has been developed by Microsoft Research (Redmond, Washington, USA) and was recently validated. ${ }^{43}$ We will use this device to make a short recording of the radial pulse wave, from which Peripheral Augmentation Index (PAIx) and CAIx will be obtained. PAIx will be calculated as (second peak SBP (SBP2) - DBP)/(first peak SBP - DBP) x 100, to yield a percentage (\%) value. From the estimated morphology of the aortic wave, by mathematical transformation specific to the wrist-worn device, CAIx will be calculated using the following formula: central augmentation pressure $\mathrm{x}$ 100/pulse pressure.

\section{Assessment of vascular structure by carotid IMT}

Carotid ultrasound will be performed by investigators trained for this purpose before starting the study, to assess carotidIMT (C-IMT). Measurements will be made of the common carotid after the examination of a $10 \mathrm{~mm}$ longitudinal section at a distance of $1 \mathrm{~cm}$ from the bifurcation, performing measurements in the proximal and in the distal wall in the lateral, anterior and posterior projections, following an axis perpendicular to the artery to discriminate two lines, one for the intima blood interface and the other for the media-adventitious interface. The measurements will be obtained with the participant lying down, with the head extended and slightly turned opposite to the examined carotid artery. Pathological intima media thickening: IMT $>0.9 \mathrm{~mm}$, or atheromatous plaque diameter greater than $1.5 \mathrm{~mm}$, or focal increase of $0.5 \mathrm{~mm}$ or $50 \%$ of the adjacent IMT. ${ }^{28}$

\section{Renal and cardiac assessment}

Kidney damage will be assessed by the estimated glomerular filtration rate using the Chronic Kidney Disease Epidemiology Collaboration (CKD-EPI) ${ }^{44}$ equation and albumin-creatinine ratio, following the criteria of the $\mathrm{ESH}^{28}$ Cardiac examination will be performed using a ECG device. ECG left ventricular hypertrophy will be defined as a Sokolow-Lyon index $>3.5 \mathrm{mV}$, or Cornell VDP $>244 \mathrm{mV} \times \mathrm{ms} 0 .{ }^{28}$

\section{Cognitive assessment}

The Montreal Cognitive Assessment (MoCA), a screening tool for dementia, validated in Spain ${ }^{45}$ and Portugal ${ }^{46}$ will be applied. The MoCA was designed as a rapid screening instrument for mild cognitive dysfunction. It assesses different cognitive domains: attention and concentration, executive functions, memory, language, visuoconstructional skills, conceptual thinking, calculations and 
orientation. Time estimated for MoCA administration is approximately 10 minutes. The total possible score is 30 points; a score of 26 or above is considered normal.

Investigators applying the different tests will be blinded to participants clinical data. All assessments will be carried out within 10 days.

\section{Statistical analysis}

Data input will be performed using the REDCap (Research Electronic Data Capture), ${ }^{47}$ with a questionnaire previously designed for the project. Normal distribution of variables will be verified using the Kolmogorov-Smirnov test. Quantitative variables will be displayed as mean $\pm \mathrm{SD}$, if normally distributed, or as median (IQR), if asymmetrically distributed, and qualitative variables will be expressed as frequencies. Analysis of difference of means between variables of two categories will be carried out using a Student's t-test or a MannWhitney $\mathrm{U}$ test, as appropriate, while qualitative variables will be analysed using a $\chi^{2}$ test. To analyse the relationship between qualitative variables of more than two categories, and quantitative variables, an analysis of variance and the least significant difference test will be used in the post hoc tests; a Kruskal-Wallis test will be used in cases where the variables are not normally distributed. The relationship of quantitative variables to each other will be tested using Pearson or Spearman correlation, as appropriate. Analysis of covariance will be performed to adjust for the variables that can affect the results as confounders. Logistic regression will be performed to evaluate the association between the study factor (gut microbial diversity) and the dependent variable (arterial stiffness), adjusted for possible confounding variables (sex, age, BMI and hypertension). A multiple linear regression will also be performed to analyse the relationship of the study factor (gut microbiota) with the variables that analyse vascular structure and function quantitatively. This regression, and all the other multivariate analyses performed, will be adjusted for the same confounding variables as the logistic regression.

Data will be analysed using the SPSS V.23.0 statistical package (SPSS Inc, Chicago, Illinois, USA). A value of $\mathrm{p}<0.05$ will be considered statistically significant. The statisticians/researchers who perform the different analyses will be blinded to participants clinical data.

\section{Quality control}

Different processes will be carried out to guarantee study data quality and thus maximise validity and reliability of measurements and results. For this purpose, field work operation manuals have been prepared. Educational leaflets will be developed to ensure correct stool collection. All of these actions will assure an adequate performance at each procedure. Monthly meetings will be held, with the investigators of both centres, to analyse the entire process, and annual reports on study progress will be prepared.

\section{Project schedule}

This project will be performed in 3years. In the first 2 years we will perform sample selection and inclusion and data collection using the previously mentioned questionnaires. During the $3^{\text {rd }}$ year, analysis and dissemination of the results will be held.

\section{ETHICS AND DISSEMINATION \\ Ethical considerations}

The study was approved by the 'Committee of ethics of research with medicines of the health area of Salamanca' on 14 December 2018 (cod. 2018-11-136) and the 'Ethics committee for health of Guimaraes' (Portugal) on 15 October 2019 (ref: 67/2019). Participants must provide informed consent, in accordance with the Declaration of Helsinki. Confidentiality of participants data will always be guaranteed, in accordance with the Regulation (European Union) 2016/679 of the European Parliament and of the Council of 27 April 2016, on the protection of natural persons with regard to the processing of personal data and on the free movement of such data, and repealing Directive 95/46/EC.

A SPIRIT (Standard Protocol Items: Recommendations for Interventional Trials) checklist is available for this protocol.

\section{Dissemination plan}

Data will be available to members of the research group and members of the Iberian Network on Arterial Structure, Central Hemodynamics and Neurocognition, following the criteria previously defined by the management team.

The research group plans to achieve rapid and widespread dissemination of results to ensure maximum visibility of this study. To this end, results of the study will be published in open-access scientific journals with peer review. At least one publication of the main results and others with the secondary results are planned. This will be complemented by the presentation of the results of the study at relevant scientific conferences and seminars, of national and international scope. Also, a doctoral thesis based on this project will be prepared. Appropriate dissemination will likewise be carried out through social networks and other media.

Patients or the public were not involved in the design, or conduct, or reporting, or dissemination plans of our research.

\section{DISCUSSION}

In recent years, there has been an increase in attention to gut microbiota richness and complexity. The detailed evaluation on the biochemical role of gut microbiome unveils its contribution to local and systemic inflammation and to the development of metabolic diseases, by both diet-dependent and independent mechanisms. ${ }^{21} 23$ 
A relation between microbiota and arterial stiffness, an early marker of vascular lesion, is expected. ${ }^{48} \mathrm{~A}$ recent study from London found an inverse association between gut microbiome diversity and arterial stiffness, in women. ${ }^{49}$ Another study, from Moscow, reported a relation between metabolic dysfunctions, gut microbiota low diversity and increased representation of opportunistic pathogens. ${ }^{50}$

We propose to analyse microbiota in patients with documented arterial stiffness. We believe that results from this study will provide novel data that will contribute to the understanding of microbiota role in the development of CVD. That knowledge may help to develop nonpharmacological approaches and strategies to prevent CVD, through lifestyle modification.

\section{Author affiliations}

${ }^{1}$ Primary Health Care Research Unit of Salamanca (APISAL), Health Service of Castilla y León (SACyL), Institute for Biomedical Research of Salamanca (IBSAL), Salamanca, Castilla y León, Spain

${ }^{2}$ Institute of Molecular and Cellular Biology of Cancer (IBMCC), Cancer Research Institute (USAL-CSIC), Institute for Biomedical Research of Salamanca (IBSAL), Salamanca, Castilla y León, Spain

${ }^{3}$ Departmente of Medicine, University of Salamanca, Salamanca, Castilla y León, Spain

${ }^{4}$ Department of Medicine, Hematology, University Hospital of Salamanca, University of Salamanca, Salamanca, Castilla y León, Spain

${ }^{5}$ Life and Health Sciences Research Institute (IICVS). School of Medicine, University of Minho, Braga, Braga, Portugal

${ }^{6}$ Department of Biomedical and Diagnostic Sciences, University of Salamanca, Salamanca, Castilla y León, Spain

${ }^{7}$ Iberian Network on Arterial Structure, School of Medicine, University of Minho, Braga, Iberian Network on Arterial Structure, Central Hemodynamics and Neurocognition, Portugal

Correction notice This article has been corrected since it first published. The provenance and peer review statement has been included.

Acknowledgements The authors are grateful to all MIVAS group investigators: Luis García-Ortiz, Pedro Guimarães-Cunha, Rita Salvado, Isabel Vila, Manuel A Gomez-Marcos, Carlos Martínez-Salgado, Jesús M Hernandez-Rivas, Rogelio Gonzalez-Sarmiento, Jesus Ma Hernandez-Sanchez, Rocio Benito, Sandra SantosMinguez, Emiliano Rodriguez-Sanchez, Maria C Patino-Alonso, Jose A MaderueloFernandez, José I Recio-Rodríguez, Jesús González-Sánchez, Susana Gonzalez Sanchez, Cristina Agudo-Conde, Cristina Lugones-Sanchez, Rosario AlonsoDomínguez, Carmela Rodriguez-Martín, Ángela de Cabo-Laso, Benigna SánchezSalgado, Natalia Sanchez-Aguadero, Sara Mora-Simón, Olaya Tamayo-Morales and $M^{a}$ Pilar Martín-Gonzalez on behalf of the Iberian Network on Arterial Structure, Central Hemodynamics and Neurocognition. We would like to especially thank Dr Alex Mira for his advice and contribution to the analysis of microbiota. We also want to thank the patient advisers who will collaborate with the study investigators disinterestedly.

Contributors LGO, PGC and RS contributed to the conception and design of the study. RS and LGO prepared the manuscript of the study protocol. CLS, CAC, ACL, MAGM, ERS, SSM, RB and JMHS contributed to the development of the study protocol. LGO and JMHR provided assistance with statistical methodology and knowledge. LGO, PGC, JMHR, MAGM and ERS provided a critical review of the manuscript. All authors have read and accepted the final version of the protocol.

Funding The project has been funded by the Carlos III Health Institute (Spain) through the Network of preventive activities and health promotion (redIAPP, RD16/0007), co-financed with European funds for regional development (FEDER) and the Autonomous Government of Castilla y León (GRS 1820/B/18; GRS 1944/B/19 and intensification programme). They played no role in the study design, analysis, reporting of results or decision to submit the manuscript for publication.

Competing interests None declared.
Patient and public involvement Patients and/or the public were not involved in the design, or conduct, or reporting, or dissemination plans of this research.

Patient consent for publication Not required.

Provenance and peer review Not commissioned; externally peer reviewed.

Open access This is an open access article distributed in accordance with the Creative Commons Attribution Non Commercial (CC BY-NC 4.0) license, which permits others to distribute, remix, adapt, build upon this work non-commercially, and license their derivative works on different terms, provided the original work is properly cited, appropriate credit is given, any changes made indicated, and the use is non-commercial. See: http://creativecommons.org/licenses/by-nc/4.0/.

ORCID iDs

Rita Salvado http://orcid.org/0000-0002-1486-3638

Manuel A Gomez-Marcos http://orcid.org/0000-0003-0133-6123

\section{REFERENCES}

1 Piepoli MF, Hoes AW, Agewall S. European guidelines on cardiovascular disease prevention in clinical practiceThe sixth joint Task force of the European Society of cardiology and other societies on cardiovascular disease prevention in clinical practice (constituted by representative. Eur Heart J 2016;2016:2315-81.

2 Vlachopoulos C, Xaplanteris P, Aboyans V, et al. The role of vascular biomarkers for primary and secondary prevention. A position paper from the European Society of cardiology Working group on peripheral circulation: endorsed by the association for research into arterial structure and physiology (artery) Society. Atherosclerosis 2015;241:507-32.

3 Vlachopoulos C, Aznaouridis K, Stefanadis C. Prediction of cardiovascular events and all-cause mortality with arterial stiffness: a systematic review and meta-analysis. J Am Coll Cardiol 2010;55:1318-27.

4 Vlachopoulos C, Aznaouridis K, Stefanadis C. Aortic stiffness for cardiovascular risk prediction. J Am Coll Cardiol 2014;63:647-9.

5 Laurent S, Marais L, Boutouyrie P. The noninvasive assessment of vascular aging. Can J Cardiol 2016;32:669-79.

6 The Reference Values for Arterial Stiffness' Collaboration. Determinants of pulse wave velocity in healthy people and in the presence of cardiovascular risk factors: 'establishing normal and reference values'. Eur Heart J 2010;31:2338-50.

7 Shirai K, Hiruta N, Song M, et al. Cardio-ankle vascular index (CAVI) as a novel indicator of arterial stiffness: theory, evidence and perspectives. J Atheroscler Thromb 2011;18:924-38.

8 Munakata M. Brachial-ankle pulse wave velocity in the measurement of arterial stiffness: recent evidence and clinical applications. Curr Hypertens Rev 2014;10:49-57.

9 Kashtanova D, Tkacheva O, Popenko A, et al. Gut microbiota and vascular biomarkers in patients without clinical cardiovascular diseases. Artery Res 2017;18:41-8.

10 Marques FZ, Mackay CR, Kaye DM. Beyond gut feelings: how the gut microbiota regulates blood pressure. Nat Rev Cardiol 2018;15:20-32.

11 Boulangé CL, Neves AL, Chilloux J, et al. Impact of the gut microbiota on inflammation, obesity, and metabolic disease. Genome Med 2016;8:42.

12 Blandino G, Inturri R, Lazzara F. Impact of gut microbiota on diabetes mellitus. Diabetes and Metabolism: Elsevier Masson, 2016: 303-15.

13 Suez J, Korem T, Zeevi D, et al. Artificial sweeteners induce glucose intolerance by altering the gut microbiota. Nature 2014;514:181-6.

14 Chassaing B, Koren O, Goodrich JK, et al. Dietary emulsifiers impact the mouse gut microbiota promoting colitis and metabolic syndrome. Nature 2015;519:92-6.

15 Schnorr SL, Candela M, Rampelli S, et al. Gut microbiome of the Hadza hunter-gatherers. Nat Commun 2014;5:3654.

16 Hung S-C, Yang T-M, Tarng D-C. SP260HIGH salt diet alters gut microbiota leading to inflammation and progression of CKD. Nephrology Dialysis Transplantation 2017;32:iii194-iii94.

17 Wu H, Tremaroli V, Bäckhed F. Linking microbiota to human diseases: a systems biology perspective. Trends Endocrinol Metab 2015;26:758-70.

18 David LA, Maurice CF, Carmody RN, et al. Diet rapidly and reproducibly alters the human gut microbiome. Nature 2014;505:559-63.

19 Wu GD, Chen J, Hoffmann C, et al. Linking long-term dietary patterns with gut microbial enterotypes. Science 2011;334:105-8.

20 Hall AB, Tolonen AC, Xavier RJ. Human genetic variation and the gut microbiome in disease. Nat Rev Genet 2017;18:690-9. 
21 Yiu JHC, Dorweiler B, Woo CW. Interaction between gut microbiota and Toll-like receptor: from immunity to metabolism. $\mathrm{J}$ Mol Med 2017;95:13-20.

22 Cox AJ, West NP, Cripps AW. Obesity, inflammation, and the gut microbiota. Lancet Diabetes Endocrinol 2015;3:207-15.

23 Qi J, You T, Li J, et al. Circulating trimethylamine N-oxide and the risk of cardiovascular diseases: a systematic review and meta-analysis of 11 prospective cohort studies. J Cell Mol Med 2018;22:185-94.

24 Tang WHW, Wang Z, Levison BS, et al. Intestinal microbial metabolism of phosphatidylcholine and cardiovascular risk. $N$ Engl J Med 2013;368:1575-84.

25 Cho CE, Taesuwan S, Malysheva OV. Trimethylamine- $N$-oxide (TMAO) response to animal source foods varies among healthy young men and is influenced by their gut microbiota composition: A randomized controlled trial. Mol Nutr Food Res 2017;61:1600324.

26 Gomez-Marcos MA, Martinez-Salgado C, Gonzalez-Sarmiento $\mathrm{R}$, et al. Association between different risk factors and vascular accelerated ageing (EVA study): study protocol for a cross-sectional, descriptive observational study. BMJ Open 2016;6:e011031.

27 Cunha PG, Cotter J, Oliveira P, et al. The Rationale/Design of the Guimarães/Vizela study. Journal of Investigative Medicine 2014;62:813-20.

28 Williams B, Mancia G, Spiering W, et al. 2018 ESC/ESH guidelines for the management of arterial hypertension. Eur Heart $J$ 2018;39:3021-104.

29 Schröder H, Fitó M, Estruch R, et al. A short screener is valid for assessing Mediterranean diet adherence among older Spanish men and women. J Nutr 2011;141:1140-5.

30 Recio-Rodriguez JI, Gómez-Marcos MA, Agudo-Conde C, et al. Evident 3 study: a randomized, controlled clinical trial to reduce inactivity and caloric intake in sedentary and overweight or obese people using a smartphone application: study protocol. Medicine 2018;97:e9633.

31 Martin-Moreno JM, Boyle P, Gorgojo L, et al. Development and validation of a food frequency questionnaire in Spain. Int J Epidemio 1993;22:512-9.

32 Pedro Moreira DS. Maria Daniel Vaz de Almeida. Validade relativa de um questionário de frequ|encia alimentar através dA comparação CoM um registo alimentar de quatro dias. Acta Médica Portuguesa 2003;16:412-20.

33 Craig CL, Marshall AL, Sjöström M, et al. International physical activity questionnaire: 12 -country reliability and validity. Med Sci Sports Exerc 2003;35:1381-95.

34 Marshall AL, Miller YD, Burton NW, et al. Measuring total and domain-specific sitting: a study of reliability and validity. Med Sci Sports Exerc 2010;42:1094-102.
35 Klindworth A, Pruesse E, Schweer T, et al. Evaluation of general 16S ribosomal RNA gene PCR primers for classical and next-generation sequencing-based diversity studies. Nucleic Acids Res 2013;41:e1.

36 Yang B, Wang Y, Qian P-Y. Sensitivity and correlation of hypervariable regions in 16S rRNA genes in phylogenetic analysis. BMC Bioinformatics 2016;17:135.

37 Gohl D, Gohl DM, MacLean A, et al. An optimized protocol for highthroughput amplicon-based microbiome profiling. Protoc Exch 2016.

38 Edgar RC, Haas BJ, Clemente JC, et al. UCHIME improves sensitivity and speed of chimera detection. Bioinformatics 2011;27:2194-200.

39 Reference Values for Arterial Stiffness' Collaboration. Determinants of pulse wave velocity in healthy people and in the presence of cardiovascular risk factors: 'establishing normal and reference values'. Eur Heart J 2010;31:2338-50.

40 Shirai K, Utino J, Otsuka K, et al. A novel blood pressureindependent arterial wall stiffness parameter; cardio-ankle vascular index (CAVI). J Atheroscler Thromb 2006;13:101-7.

41 Yamashina A, Tomiyama H, Takeda K, et al. Validity, reproducibility, and clinical significance of noninvasive brachial-ankle pulse wave velocity measurement. Hypertens Res 2002;25:359-64.

42 Kawai T, Ohishi M, Onishi M, et al. Cut-Off value of brachial-ankle pulse wave velocity to predict cardiovascular disease in hypertensive patients: a cohort study. J Atheroscler Thromb 2013;20:391-400.

43 García-Ortiz L, Recio-Rodríguez JI, Agudo-Conde C, et al. Noninvasive validation of central and peripheral augmentation index estimated by a novel wrist-worn tonometer. J Hypertens 2018;36:2204-14.

44 Levey AS, Stevens LA, Schmid CH, et al. A new equation to estimate glomerular filtration rate. Ann Intern Med 2009;150:604-12.

45 Delgado C, Araneda A, Behrens MI. Validation of the Spanishlanguage version of the Montreal cognitive assessment test in adults older than 60 years. Neurologia 2019;34:376-85.

46 Freitas S, Simões MR, Alves L, et al. Montreal cognitive assessment (MoCA): validation study for vascular dementia. J Int Neuropsychol Soc 2012;18:1031-40.

47 Harris PA, Taylor R, Thielke R, et al. Research electronic data capture (REDCap)--a metadata-driven methodology and workflow process for providing translational research informatics support. J Biomed Inform 2009;42:377-81.

48 van den Munckhof ICL, Kurilshikov A, Ter Horst R, et al. Role of gut microbiota in chronic low-grade inflammation as potential driver for atherosclerotic cardiovascular disease: a systematic review of human studies. Obes Rev 2018;19:1719-34.

49 Menni C, Lin C, Cecelja M, et al. Gut microbial diversity is associated with lower arterial stiffness in women. Eur Heart J 2018;39:2390-7.

50 Kashtanova DA, Tkacheva ON, Doudinskaya EN, et al. Gut microbiota in patients with different metabolic statuses: Moscow study. Microorganisms 2018;6. doi:10.3390/microorganisms6040098 